\title{
PRECURSORS TO INTERSTELLAR SHOCKS OF SOLAR ORIGIN
}

\author{
D. A. Gurnetr ${ }^{1}$, W. S. Kurth ${ }^{1}$, E. C. Stone $^{2}$, A. C. Cummings ${ }^{2}$, S. M. Krimigis ${ }^{3}$, R. B. Decker ${ }^{3}$, \\ N. F. Ness ${ }^{4}$, AND L. F. Burlaga ${ }^{5}$ \\ ${ }^{1}$ University of Iowa, Department of Physics and Astronomy, Iowa City, IA 52242, USA; donald-gurnett@uiowa.edu \\ ${ }^{2}$ California Institute of Technology, 1200 East California Boulevard, Pasadena, CA 91125, USA \\ ${ }^{3}$ Applied Physics Laboratory/JHU, 11100 Johns Hopkins Road, Laurel, MD 20723, USA \\ ${ }_{5}^{4}$ Catholic University of America, 620 Michigan Avenue NE, Washington, DC 20064, USA \\ ${ }^{5}$ NASA Goddard Space Flight Center, 8800 Greenbelt Road, Greenbelt, MD 20771, USA \\ Received 2015 April 29; accepted 2015 July 14; published 2015 August 14
}

\begin{abstract}
On or about 2012 August 25, the Voyager 1 spacecraft crossed the heliopause into the nearby interstellar plasma. In the nearly three years that the spacecraft has been in interstellar space, three notable particle and field disturbances have been observed, each apparently associated with a shock wave propagating outward from the Sun. Here, we present a detailed analysis of the third and most impressive of these disturbances, with brief comparisons to the two previous events, both of which have been previously reported. The shock responsible for the third event was first detected on 2014 February 17 by the onset of narrowband radio emissions from the approaching shock, followed on 2014 May 13 by the abrupt appearance of intense electron plasma oscillations generated by electrons streaming outward ahead of the shock. Finally, the shock arrived on 2014 August 25, as indicated by a jump in the magnetic field strength and the plasma density. Various disturbances in the intensity and anisotropy of galactic cosmic rays were also observed ahead of the shock, some of which are believed to be caused by the reflection and acceleration of cosmic rays by the magnetic field jump at the shock, and/or by interactions with upstream plasma waves. Comparisons to the two previous weaker events show somewhat similar precursor effects, although differing in certain details. Many of these effects are very similar to those observed in the region called the "foreshock" that occurs upstream of planetary bow shocks, only on a vastly larger spatial scale.
\end{abstract}

Key words: cosmic rays - local interstellar matter - shock waves - Sun: heliosphere

\section{INTRODUCTION}

For over 50 years scientists have postulated a boundary, called the heliopause, which separates the heliospheric plasma from the interstellar plasma (Axford 1990; Davis 1955; Parker 1963). After a flight of 35 years the Voyager 1 spacecraft finally reached the vicinity of the heliopause on 2012 July 27 , and after passing through a complex boundary layer region crossed into the interstellar plasma on 2012 August 25 at a heliocentric radial distance of 121.6 Astronomical Units (AU). This crossing is marked by the vertical line labeled "Heliopause" in Figure 1. In the data from the cosmic ray (CRS) and low energy charged particle (LECP) instruments (Krimigis et al. 2013; Stone et al. 2013; Webber \& McDonald 2013), the heliopause was characterized by a large decrease in the anomalous cosmic ray intensity and an increase in the galactic cosmic ray intensity, see Figures 1(a) and (b), and by an increase in the magnetic field strength as measured by the magnetometer (MAG) instrument (Burlaga et al. 2013a), see Figure 1(c). Initially, there was some uncertainty as to whether these changes marked the crossing of the heliopause because there was almost no change in the magnetic field direction. However, about two months later, on 2012 October 23, the plasma wave (PWS) instrument detected the first of three electron plasma oscillation events (Gurnett et al. 2013), all three of which can be seen in Figure 1(d). Plasma oscillations of this type are known to be produced ahead of shocks propagating outward from energetic events at the Sun, and occur at the electron plasma frequency, $f_{\mathrm{p}}=8980 \sqrt{ } n_{\mathrm{e}} \mathrm{Hz}$, where $n_{\mathrm{e}}$ is the electron density in $\mathrm{cm}^{-3}$. The electron densities inferred from the frequency of the plasma oscillations showed that the plasma density had increased markedly, from the very low densities, $\sim 0.002 \mathrm{~cm}^{-3}$, characteristic of the outer region of the heliosphere (Richardson et al. 2008) to the much higher densities, $0.05-0.22 \mathrm{~cm}^{-3}$, expected in the local interstellar medium (Frisch et al. 2011). The density measurements from the PWS provided the confirming evidence that the spacecraft had crossed into the interstellar plasma around the time of the major changes in the energetic particle intensities, i.e., 2012 August 25. The white dashed lines in Figure 1(d) show that in the first few months after the crossing, the density of the interstellar plasma increased from about 0.06 to $0.08 \mathrm{~cm}^{-3}$. We called this region the "density ramp." After this initial rapid increase, the density has increased only slightly, to about $0.09-0.11 \mathrm{~cm}^{-3}$ in the most recent 2014 data. It is interesting to note that the density ramp was first detected by remote radio measurements from Voyagers 1 and 2 over 20 years ago (Gurnett et al. 1993). For a further discussion of the origin of the density ramp, specifically the "plasma depletion model," see Fuselier \& Cairns (2013).

As discussed above, three plasma oscillation events have been observed in the interstellar plasma, the first in late-2012, the second in mid-2013, and the third lasting most of 2014, all of which are believed to be associated with shock waves propagating outward from energetic solar events, i.e., coronal mass ejections (Burlaga et al. 2013b; Gurnett et al. 2013; Kurth et al. 2014). Based on early studies of terrestrial bow shocks (Scarf et al. 1971; Anderson et al. 1981; Gurnett et al. 1981; Fitzenreiter et al. 1984; Gurnett 1985), the origin of these shock-related electron plasma oscillations is now well understood, and we shall apply this understanding to the interpretation of the interstellar case. For the terrestrial bow shock, it is known that electrons accelerated by the shock escape along magnetic field lines ahead of the shock and drive electron 




Figure 1. Overview of key Voyager 1 measurements near and beyond the heliopause. (a) The counting rate from the cosmic ray (CRS) instrument in two energy ranges, mainly protons with energies $>70 \mathrm{MeV}$ (red) and protons with energies 7-60 MeV (blue). The last of several closely spaced encounters with the heliopause is indicated at the top on 2012 August 25. (b) The counting rate from the low energy charged particle (LECP) instrument for galactic cosmic rays (GCRs), mainly protons, with energies $>211 \mathrm{MeV}$ propagating parallel (red) and perpendicular (gray) to the magnetic field. (c) The magnetic field strength in nano Tesla (nT) from the magnetometer (MAG) instrument. (d) A color-coded high-resolution spectrogram of the wideband electric field spectral densities detected by the plasma wave (PWS) instrument (red is intense, dark blue is least intense). The frequency is on the left, and the corresponding electron density $n_{\mathrm{e}}$ is on the right, assuming that the emissions are at the electron plasma frequency, $f_{\mathrm{p}}=8980 \sqrt{ } n_{\mathrm{e}} \mathrm{Hz}$, where $n_{\mathrm{e}}$ is in $\mathrm{cm}^{-3}$.

plasma oscillations (also called Langmuir waves) via a beamplasma instability. The crucial process that assures the escaping upstream electrons have the proper bump-on-tail velocity distribution to drive the beam-plasma instability is spatial velocity filtering, a concept first proposed by Filbert \& Kellogg (1979), and later refined by Cairns (1987). For a shock front that curves away from the incoming plasma flow, the bump-ontail velocity distribution is due to the existence of a threshold velocity below which electrons cannot reach a given point upstream of the shock. The region accessible to such beams is often called the foreshock, or more precisely the electron foreshock, since it is electrons escaping from the shock that dominate this region, see Figure 2. The electron beam energies that drive electron plasma oscillations are typically relatively low, from a few hundred $\mathrm{eV}$ to several $\mathrm{keV}$. Because the electron beam velocities are much less than the speed of light, the leading edge of the electron foreshock is located well behind the magnetic field line tangent to the shock. An ion 


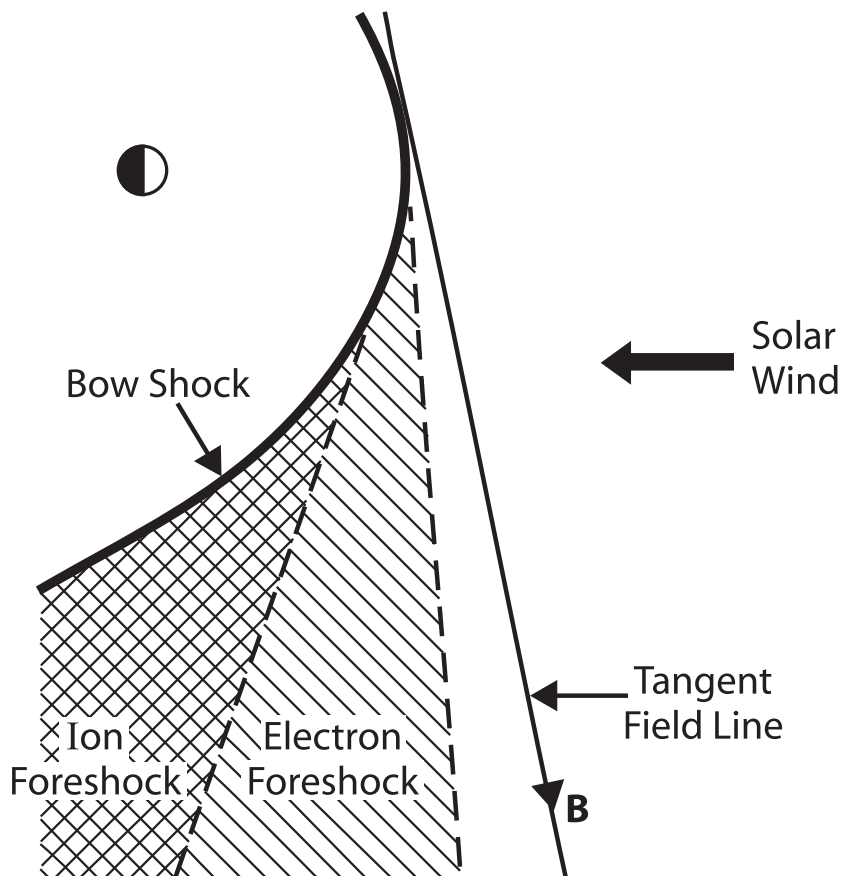

Figure 2. Foreshock geometry commonly used in the analysis of the terrestrial bow shock. Electrons streaming outward along magnetic field lines linked to the bow shock are restricted to the region marked "electron foreshock." Ions are restricted to a similar region called the "ion foreshock," which is well behind the electron foreshock because of the slower velocities of ions accelerated at the shock. The magnetic field line tangent to the shock is called the "tangent field line." The electron foreshock must lie behind the tangent field line, because only a particle with an infinite velocity could be on this field line. Within the electron foreshock, time-of-flight considerations lead to a bump-on-tail velocity distribution (i.e., a beam) that is exactly what is required to drive electron plasma oscillations. Electron plasma oscillations are usually most intense near the upstream edge of the electron foreshock where the beam velocity is the highest.

foreshock also exists that is even farther behind the electron foreshock due to the generally lower velocities of ions escaping from the shock.

To apply the results from the terrestrial bow shock to a shock propagating through the interstellar medium one must recognize that the relevant reference frames are somewhat different. For the terrestrial case the shock is usually viewed as being stationary, with the solar wind approaching at a uniform velocity from the Sun, whereas for the interstellar case the shock is propagating radially outward from the Sun through the interstellar plasma, which to a first approximation can be regarded as stationary. Although the scale sizes are different, in both cases the shock front is curved away from the incident plasma flow. Therefore, once the change in the reference frame is taken into account, one can see that locally the geometries are essentially the same. Since the interstellar magnetic field direction is nearly constant (Burlaga \& Ness 2014), whereas the solar wind magnetic field direction is often highly variable, the analysis of effects upstream of an interstellar shock is potentially even simpler than for the terrestrial bow shock.

In the following section we will examine how the above concepts apply to shocks in the interstellar medium. As we will see, despite the vastly different spatial scales, more than a factor of ten thousand, there are many very close similarities and also some unexpected effects that have no known counterpart in the terrestrial bow shock.

\section{THE 2014 EVENT}

An expanded time-scale plot showing details of the 2014 electron plasma oscillation event is given in Figure 3. The first evidence of the shock responsible for this event was the detection of a very weak narrowband emission in the PWS wideband spectrogram at about $2.7 \mathrm{kHz}$ on day 48 , see Figure 3(a). About three months later, on day 134 this emission suddenly became very intense and spiky, so intense that it briefly causes distortion in the PWS wideband receiver, as indicated by a broadening of the spectrum for a few days after the onset. The abrupt intensification and spiky variations are especially clear in the $3.11 \mathrm{kHz}$ channel of the onboard 16channel spectrum analyzer, see Figure 3(b). Such intense spiky narrowband emissions are typical of electrostatic electron plasma oscillations in the terrestrial foreshock at the electron plasma frequency. In the context of the terrestrial observations, the abrupt intensification on day 134 corresponds to the leading edge of the electron foreshock. It should be noted that although the electron plasma oscillations are almost certainly driven by an electron beam, no evidence of such a beam has been found in the Voyager 1 data. This is because the Voyager 1 plasma (PLS) instrument, which could possibly have detected such beams, failed in 1980, and the energy range of the LECP, >28 $\mathrm{keV}$, does not extend low enough to reliably detect these beams.

The narrowband emissions observed ahead of the intense plasma oscillations appear to have a close analog with terrestrial observations. At Earth narrowband radio emissions are commonly observed ahead of the shock at either $f_{\mathrm{p}}$ or $2 f_{\mathrm{p}}$, or both, and are known to be generated by nonlinear mode conversion from electron plasma oscillations in the foreshock (Hoang et al. 1981; Burgess et al. 1987; Kuncic et al. 2002; Kuncic \& Cairns 2005). Also, Type II solar radio bursts are produced by the same mode conversion processes in the solar corona and in the solar wind upstream of interplanetary shocks (Reiner et al. 1998a, 1998b; Bale et al. 1999; Cairns et al. 2003). From these comparisons we conclude that the weak narrowband emissions observed from day 48 to day 134 are radio emissions (i.e., electromagnetic radiation), and that these emissions are produced via nonlinear mode conversion at or near $f_{\mathrm{p}}$ by the strong electrostatic electron plasma oscillations near the leading edge of the foreshock. That the radio emission is primarily at $f_{\mathrm{p}}$ and not at $2 f_{\mathrm{p}}$ is consistent with the predictions of Cairns \& Zank $(2001,2002)$ for the parameters that exist in the interstellar medium. Because the nonlinear mode conversion process generates radio emission at the difference of the frequencies of two interacting waves, the fact that radio emission frequency is at almost the same as the frequency of the plasma oscillations (within $\sim 100 \mathrm{~Hz}$ ) implies that a second interacting wave must be present at a very low frequency, probably much less than $100 \mathrm{~Hz}$. Although the frequency range of the PWS goes down to $10 \mathrm{~Hz}$, we have not been able to detect these waves, possibly because of the large amount of spacecraft-generated interference at these low frequencies.

A little over three months after the onset of the intense plasma oscillations, on day 237,2014 , the shock finally arrived at the spacecraft. The shock can be clearly identified by the jump in the magnetic field strength marked "shock" in Figure 3(c), and by a corresponding jump in the plasma density, as inferred from the jump in the low-frequency cutoff of the emission spectrum marked "shock" in Figure 3(a). The 




Figure 3. Details of the 2014 event. (a) An expanded frequency-time spectrogram of the electric field intensity. The spectrums are obtained by Fourier transforming the electric field waveforms from the PWS wideband receiver. Because the wideband receiver has an automatic gain control, the spectral intensities are only relative and do not provide absolute measurements of electric field strength. (b) The electric field intensity from the $3.11 \mathrm{kHz}$ channel of the PWS on board the 16-channel spectrum analyzer. Since this channel is not centered on the emission frequency, which is about $2.7 \mathrm{kHz} \pm 100 \mathrm{~Hz}$, we estimate from the filter response that the electric field intensities are about a factor of 10 greater than shown. (c) One-day averages of the magnetic field strength from the magnetometer (MAG). (d) The counting rate of electrons with energies of 5-13 MeV from the cosmic ray (CRS) instrument. (e) The omnidirectional counting rate from the G1 channel (mainly protons with $>20 \mathrm{MeV}$ ) of the cosmic ray (CRS) instrument. Note the greatly expanded counting rate scale. (f) Three-day averages of the counting rates of galactic cosmic ray protons with energies $>211 \mathrm{MeV}$ from the low energy charged particle (LECP) instrument. The measurements are made in eight $45^{\circ}$ angular viewing directions that are all very nearly in the (R, T) plane, where $\mathrm{R}$ is radially outward from the Sun, and $\mathrm{T}$ is parallel to the solar equator and positive in the direction of the Sun's rotation, see the diagram in the lower left corner. Gray points are the averages of the S1 and S5 sectors, green points are the averages of the S2 and S6 sectors, and blue points are the averages of the S3 and S7 sectors. The sectors are numbered starting at 1 in the first $0^{\circ}-45^{\circ}$ sector, where the angle is measured starting in the $+\mathrm{R}$ direction and measured positive toward the $+\mathrm{T}$ direction. 


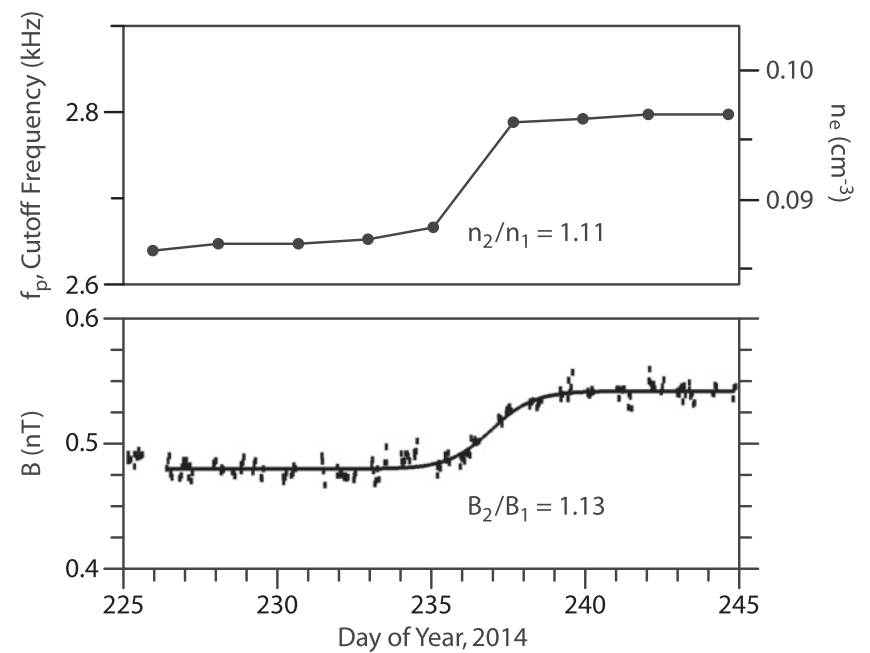

Figure 4. Expanded scale plots of the electron density and magnetic field strength near the 2014 shock. (a) The frequency of the low-frequency cutoff of the plasma wave emission spectrum is given on the left, and the corresponding electron density, $n_{\mathrm{e}}$, is given on the right, assuming that the cutoff is at the local electron plasma frequency, $f_{\mathrm{p}}$. (b) The magnetic field strength from the magnetometer. Note the very good agreement of the jumps in the plasma density and the magnetic field strength.

relationship between the jump in the low-frequency cutoff and the inferred jump in the plasma density probably needs further explanation. Note from Figure 3(b) that near the shock the spiky intensity variations characteristic of the electron plasma oscillations have almost completely disappeared and the emission has become very smooth. Such smooth emissions are typical of radio emissions. This change in the character of the emission leads us to believe that in this region the spectrum is due mainly to radio emissions generated by the mode conversion process. Since radio emissions cannot propagate at frequencies below the electron plasma frequency, the lowfrequency cutoff of these emissions must be at the electron plasma frequency, which then gives the local electron density via the previously given equation for $f_{\mathrm{p}}$.

It is interesting to do a detailed analysis of the electron density and the magnetic field jumps at the shock. Figure 4 shows a comparison of the electron density, $n_{\mathrm{e}}$, computed from the low-frequency cutoff of the radio emission, and the magnetic field strength, $B$, as measured by the magnetometer. As can be seen, the timing of the jump in the plasma density agrees very well with the jump in the magnetic field strength. Although not shown, there is no discernible change in the magnetic field direction at the shock. The absence of a change in the magnetic field direction means that the shock must be a quasi-perpendicular shock. Since for a quasi-perpendicular shock the plasma and the magnetic field are compressed by the same amount, the ratio of the electron densities across the shock must be the same as the ratio of magnetic field strengths across the shock. For the electron density the average densities before and after the shock are 0.0873 and $0.0968 \mathrm{~cm}^{-3}$, respectively, which gives a density ratio of $n_{2} / n_{1}=1.11$. For the magnetic field the average magnetic field strengths before and after the shock are 0.480 and $0.541 \mathrm{nT}$, respectively, which gives a magnetic field strength ratio of $B_{2} / B_{1}=1.13$. This good agreement provides strong evidence that the discontinuity is indeed a perpendicular shock. Note that the spatial scale of the shock transition region, $\sim 8600 \mathrm{~km}$ for a shock speed of $40 \mathrm{~km} \mathrm{~s}^{-1}$, is rather large compared to other shocks observed in

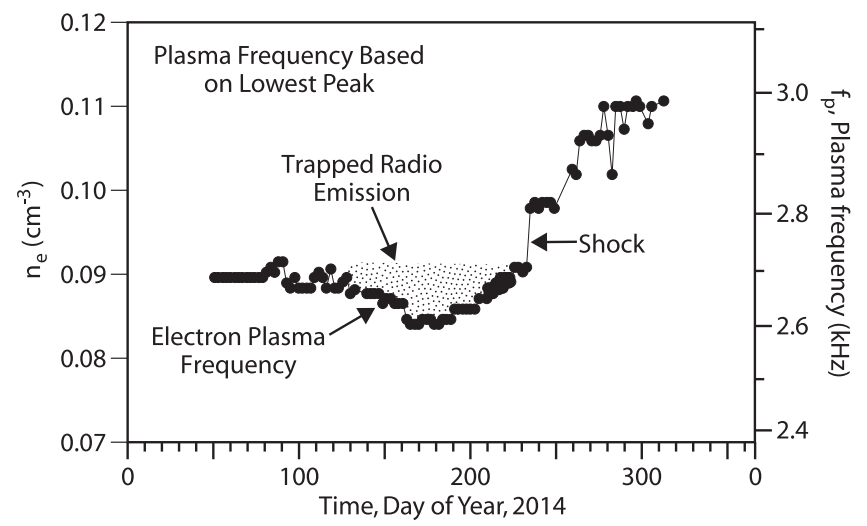

Figure 5. Plot of the electron plasma frequency and plasma density for the 2014 event. In this case we have taken the lowest peak in the emission spectrum to be representative of the electron plasma frequency. Note the parabola-shaped minimum in the plasma frequency from near the onset of the plasma oscillations on day 134 to the shock on day 237 . The emission spectrum in this region is notably broader than before or after this interval. This relatively broad spectrum consists at least in part of radio emissions trapped in a parabola-shaped density cavity between the leading edge of the foreshock (at day 134) and the shock (at day 237).

the outer regions of the heliosphere. Also, the transition is very smooth and laminar, much different than the solar wind termination shock which was very turbulent (Burlaga et al. 2005). These same distinctions have been previously noted by Burlaga et al. (2013b) in their analysis of the perpendicular shock associated with the 2012 plasma oscillation event.

From the wideband spectrogram in Figure 3(a) one can see that in the interval from the onset of the intense plasma oscillations on day 134 to the arrival of the shock on day 237 , the emission spectrum has a shallow parabola-shaped depression in the low-frequency cutoff. The frequency of this cutoff starts at about $2.70 \mathrm{kHz}$ on day 134 , reaches a minimum of about $2.55 \mathrm{kHz}$ around day 170 , and ends at about $2.65 \mathrm{kHz}$ on day 237, just before the shock. The depression in the lowfrequency cutoff and the enhanced intensities in this region suggest that the radio waves are trapped in a shallow density minimum just ahead of the shock. This feature is illustrated in Figure 5, along with a plot of the electron plasma frequency. After the passage of the shock, one can see that the plasma frequency starts to steadily increase, from about $2.79 \mathrm{kHz}$ on day 238 to about $3.00 \mathrm{kHz}$ on day 310 , after which it can no longer be detected. In principle, this emission could be either plasma oscillations or radio emissions. However, from their narrow bandwidth we believe they are locally generated electron plasma oscillations. If they are, then the density would vary from $0.097 \mathrm{~cm}^{-3}$ immediately after the shock to $0.112 \mathrm{~cm}^{-3}$ at the end of the event. We interpret this density increase as being due to the pressure pulse driving the shock.

In addition to the upstream plasma wave emissions, Figure 3 shows that the cosmic ray intensities have some very interesting variations, especially when the counting rates are plotted on a greatly expanded scale. Figure 3(d) shows the counting rates for electrons with energies of 5-13 MeV from the CRS instrument, and Figure 3(e) shows the counting rate of mainly protons with energies $>20 \mathrm{MeV}$ from CRS. Figure 3(f) shows the counting rate of mainly protons with energies $>211$ $\mathrm{MeV}$ from the LECP instrument. Two main effects can be seen from these plots: (1) a small but very clear flat-topped enhancement in the cosmic ray electron and proton intensities from day 111 to day 134 , as marked by the vertical dashed 
lines; and (2) a broad V-shaped depression in the energetic proton intensities that starts near the beginning of the plot and terminates just before the arrival of the shock (also see Figure 1(b)). Note that the enhanced CRS electron and proton intensities have very similar intensity variations with no noticeable time delays. The CRS instrument gives no information on the pitch-angle distribution of these particles. However, the LECP instrument, which makes measurements in eight angular sectors near the $(\mathrm{R}, \mathrm{T})$ plane, shows that although the small nearly flat-topped enhancement in the intensities from day 111 to day 134 is most notable in the S2-S6 sectors (green), it is still detectable in all the other sectors, indicating that the enhancement extends over a broad range of pitch angles. Even though we focus on the flat-topped feature from day 111 to day 134, note from the hatching that at lower intensities the enhancement appears to start well before day 111 and ends well after day 134. Also, note that the beginning of the rapid intensity decrease on day 134 corresponds almost exactly with the onset of the intense upstream electron plasma oscillations. In both the CRS and LECP data one is given the strong impression that the flat-topped enhancement (hatched) appears to be superposed on a broad V-shaped depression in the counting rate that starts near the beginning of the plot and ends on day 235, just before the shock, after which the counting rate is nearly constant. This same V-shaped depression in the counting rate is also shown by the dark-gray dotted line in Figure 1(b), which is for particles moving nearly perpendicular to the magnetic field, i.e., LECP sectors S1-S5. No similar depression is seen for particles moving parallel to the magnetic field (red triangles), i.e., sectors S3-S7. These observations show that the $\mathrm{V}$-shaped depression is limited to ions with pitch angles near $90^{\circ}$.

\section{COMPARISON TO THE 2012 AND 2013 EVENTS}

It is instructive next to compare the 2014 event to the previous events in 2012 and 2013, see Figure 1(d). The 2012 event has been analyzed in some detail by Burlaga et al. (2013b) and consists of very weak electron plasma oscillations followed by a very well-defined jump in the magnetic field indicative of a quasi-perpendicular shock, very similar to the 2014 event. However, for this event no radio emission was detected upstream of the shock. Although there were small changes in the directionally averaged CRS cosmic ray intensities, there was no clear evidence of a flat-topped enhancement comparable to that observed from days 111 to 134 in the 2014 event. However, there is one feature in both the CRS and LECP data that has a close similarity to the 2014 event, and that is the V-shaped depression in the intensity of particles moving perpendicular to the magnetic field. This feature is shown by the dark-gray dotted line in Figure 1(b). The depression in the perpendicular intensity starts near the heliopause and remains relatively constant until about day 297 , 2012. At this point the perpendicular intensity starts to increase rapidly, eventually reaching the intensity of particles moving parallel to the magnetic field (red triangles) at the shock on day 335,2012 . This trend toward isotropy at the shock is very similar to the cosmic ray pitch-angle variations observed for the 2014 event.

The comparison with the somewhat stronger event in 2013 has proven to be even more interesting. An overview of the plasma wave, magnetic field, and energetic particle measurements for this event is shown in Figure 6. For this event the electron plasma oscillations have a very sharp onset on about day 99. Following our earlier comparisons with the terrestrial bow shock, we would interpret this onset as the leading edge of the electron foreshock. Again, as in the 2012 plasma oscillation event, there is no detectable radio emission propagating outward from the electron foreshock, probably because the electron plasma oscillations were not sufficiently intense. Although we are confident that this event is caused by a shock propagating outward from the Sun (Kurth et al. 2014), there is no clear jump in the magnetic field strength that can be unambiguously identified as a shock, see Figure 6(c). However, there is again a remarkable relationship between the onset of the plasma oscillations and a small flat-topped enhancement (hatched) in the cosmic ray electron and proton intensities, which can be seen in the CRS counting rates and the LECP counting rates from day 80 to day 94, see Figures 6(d) and (e). Note again that the enhancement in the LECP intensities occurs over a broad range of pitch angles, and that the termination of the enhancement on day 94 occurs very close to the onset of the electron plasma oscillations, all very similar to the 2014 event. Also, there is a broad V-shaped depression in the CRS counting rates and the LECP counting rates in the perpendicular sector, S1-S5, that begins near day 350, 2012, and ends on about day 225,2013 . These variations and their qualitative similarity to the 2014 event provide strong supporting evidence that these are foreshock effects linked remotely to a shock propagating outward from the Sun. From the rapid increase in the CRS counting rate and the corresponding increasing counting rate in the perpendicular, S1-S5, sector of LECP, we suspect that the shock may be located around day 210-215, as indicated by the vertical arrow marked "Shock?." The absence of a local shock signature is probably because the spacecraft was near, but just beyond, the outer edge of the shock front.

\section{SUMMARY AND INTERPRETATION}

Here we have described various precursor effects associated with interstellar shocks that originate from the Sun. If we take the 2014 event to be the prototypical event, these effects include: (1) upstream radio emissions and electron plasma oscillations at or near the electron plasma frequency; (2) a flattopped enhancement of about $1.5 \%$ in the cosmic ray electron and ion intensities over a broad range of pitch angles just ahead of the onset of the intense electron plasma oscillations; (3) a $\mathrm{V}$-shaped depression of about $2 \%$ in the cosmic ray ion intensities at pitch angles near $90^{\circ}$ that extends over a broad region ahead of the shock and becomes isotropic at the shock; and (4) a shallow parabola-shaped density depression of about $8 \%$ in the region between the onset of the electron plasma oscillations and the shock.

The challenge we now have is to integrate all of these effects into a single coherent physical picture. As has been described earlier, the observations of upstream radio emission and plasma oscillations closely follow the electron foreshock model first developed by Filbert \& Kellogg (1979) for the terrestrial bow shock, a model that more recently has also been applied to interplanetary shocks (Bale et al. 1999). In the interstellar version of this model, electrons accelerated at the shock stream outward into the foreshock where they produce electron plasma oscillations at $f_{\mathrm{p}}$ via a beam-plasma instability, as shown in Figure 7. The electron plasma oscillations are typically most intense near the leading edge of the foreshock where the electron beam energies are the highest. These electron plasma 



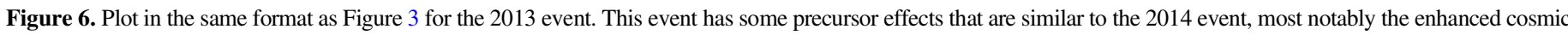

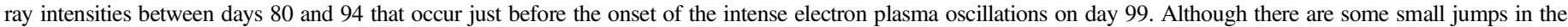
magnetic field strength, it is not clear where the shock is located or if it reached the spacecraft, even though the spacecraft clearly passes into the foreshock.

oscillations produce escaping radio emissions at or near $f_{\mathrm{p}}$ via a nonlinear mode-conversion process. According to the theory for nonlinear wave-wave interactions, this mode-conversion process requires the presence of a third wave at a frequency that is at the difference between the radio emission frequency and the electron plasma frequency (Cairns \& Robinson 1992). Since the radio emission frequency is almost the same as the frequency of the electron plasma oscillations, the third wave must be at a very low frequency, probably well below $100 \mathrm{~Hz}$, too low to be detected by the plasma wave instrument and too 


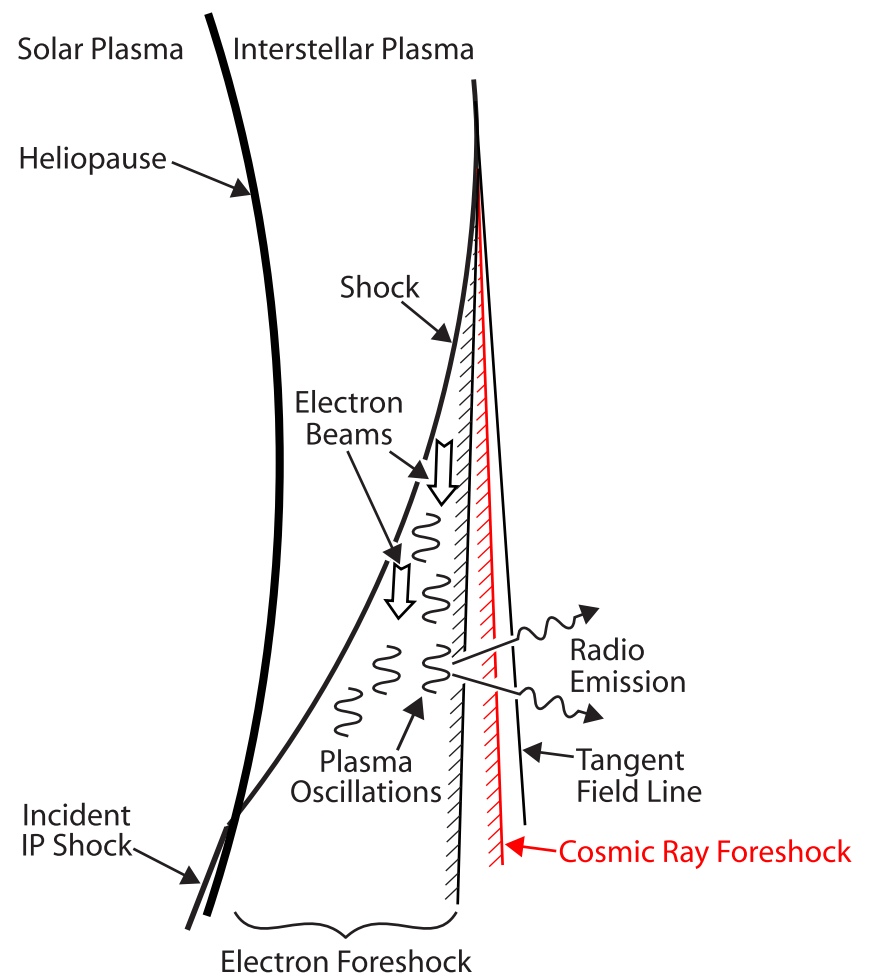

Figure 7. Foreshock model for an interplanetary shock propagating outward from the Sun. As with a planetary shock, the electron plasma oscillations are produced by electron beams streaming outward ahead of the shock along magnetic field lines connected to the shock (i.e., the electron foreshock), with the most intense emissions near the leading edge of the foreshock (black hatching). Similarly, perturbations in the cosmic ray intensities caused by their interaction with the shock are confined to a cosmic ray "foreshock," the exact leading edge of which depends on their energy. Because the cosmic rays have a considerably higher speed than electrons streaming outward from the shock, the leading edge of the cosmic ray foreshock lies ahead of the electron foreshock, but still behind the magnetic field line tangent to the shock.

weak to be observed by the magnetometer. For wave measurements the magnetometer has a relatively high noise level, 0.005 nT (Burlaga et al. 2013b). Low-frequency waves that could be involved in the mode conversion process could either be continually present or driven by the electron beam. Lower hybrid waves driven by pickup ions from interstellar hydrogen (Cairns \& Zank 2002) are an example of waves that might be continually present. Waves driven by the electron beam could include those driven directly by the beam, such as whistler-mode emissions (Sauer \& Sydora 2012), or waves driven indirectly via the mode conversion process, such as ion acoustic waves (Cairns \& Robinson 1992).

Although the radio emissions and plasma oscillations are well understood in terms of the foreshock model, the upstream effects observed in the cosmic rays have no known analog at planetary bow shocks or interplanetary shocks. To explore the possible mechanisms by which the shock could affect the cosmic rays, we start by estimating a typical gyro-radius for the ions detected by CRS and LECP. For a $200 \mathrm{MeV}$ proton moving in a $0.5 \mathrm{nT}$ magnetic field the gyro radius is $\sim 0.03 \mathrm{AU}$, much smaller than the likely radius of the shock front, which is probably many tens of AU. For the cosmic ray electrons, the gyro radii would be much smaller. For such small gyro-radii the particles can be thought of as moving almost exactly along the magnetic field lines in the plasma frame of reference. As soon as the spacecraft crosses the tangent magnetic field line, perturbations in the cosmic ray intensity caused by the interactions with the shock can propagate both forward and backward along the magnetic field line. Whether these perturbations reach the spacecraft or not then depends on time-of-flight considerations, just as in the foreshock model of Filbert \& Kellogg. Following this analogy, in the frame of reference of the shock we find it convenient to think of the cosmic ray perturbations as being confined within a region that we will call the "cosmic ray foreshock," just as electrons accelerated by the shock are confined within the "electron foreshock." For the cosmic ray ion energies considered here, $\sim 20-200 \mathrm{MeV}$, it is easily verified that these particles have speeds on the order of $20 \%-60 \%$ of the speed of light, and very close to the speed of light for the electrons. These speeds are much greater, by a factor of ten or more, than the typical electron velocities that are responsible for the electron plasma oscillations. Thus, the leading edge of the cosmic ray foreshock must lie well ahead of the electron foreshock and very near the tangent magnetic field line, as shown in Figure 7. This geometric concept explains why the onset of the cosmic ray enhancement on day 111 for the 2014 event is located ahead of the electron plasma oscillations. The same conclusion also applies to the 2013 event. The fact that the cosmic ray perturbations occur in a restricted region near the tangent field line shows that the primary interaction with the shock occurs when the magnetic field is very nearly perpendicular to the shock normal, i.e., the quasi-perpendicular region.

To investigate the origin of the cosmic ray disturbances, we next consider two types of interactions: (1) magnetic reflection and acceleration caused by the jump in the magnetic field at the shock; and (2) pitch-angle scattering and/or acceleration by the plasma wave electric fields upstream of the shock. First, we focus on the possibility that the flat-topped intensity enhancement of the cosmic ray intensities observed from days 111 to 134 during the 2014 event is caused by cosmic rays reflecting from the shock. In fact, this mechanism has already been suggested by Jokipii \& Kota (2014) to explain the enhancement observed during the 2013 event. In their model, which assumes conservation of the first adiabatic invariant, they show that cosmic rays gain energy by magnetic reflection from the shock. The energy gain occurs because in the rest frame of the upstream plasma the reflection point at the shock moves along the magnetic field line toward the spacecraft, thereby causing the incident ion to gain energy during the reflection process. Jokipii \& Kota (2014) did not discuss angular distributions of the reflected particles. We note, however, that in the absence of scattering, particles that gain the most energy by reflection at a shock would arrive at the spacecraft not as a broad angular distribution covering outgoing pitch angles $0^{\circ}-90^{\circ}$, but rather with pitch angles lying in a well-defined range that depends on particle speed and shock parameters, e.g., Decker (1983). This pitch angle range is too limited to explain the relatively broad range of ion pitch angles observed by the LECP for the 2014 event, see Figure 3(e). The same is true for the 2013 event, see Figure 6(e). Thus, although the shock reflection model provides a seemingly very attractive model for explaining the enhancement of cosmic ray ion intensities upstream of the shock, it is unlikely to be able to reproduce the observed broad angular distribution of these particles. Therefore, we conclude that either the reflected particles are somehow being scattered in pitch angle as they move from the shock to the spacecraft, or 


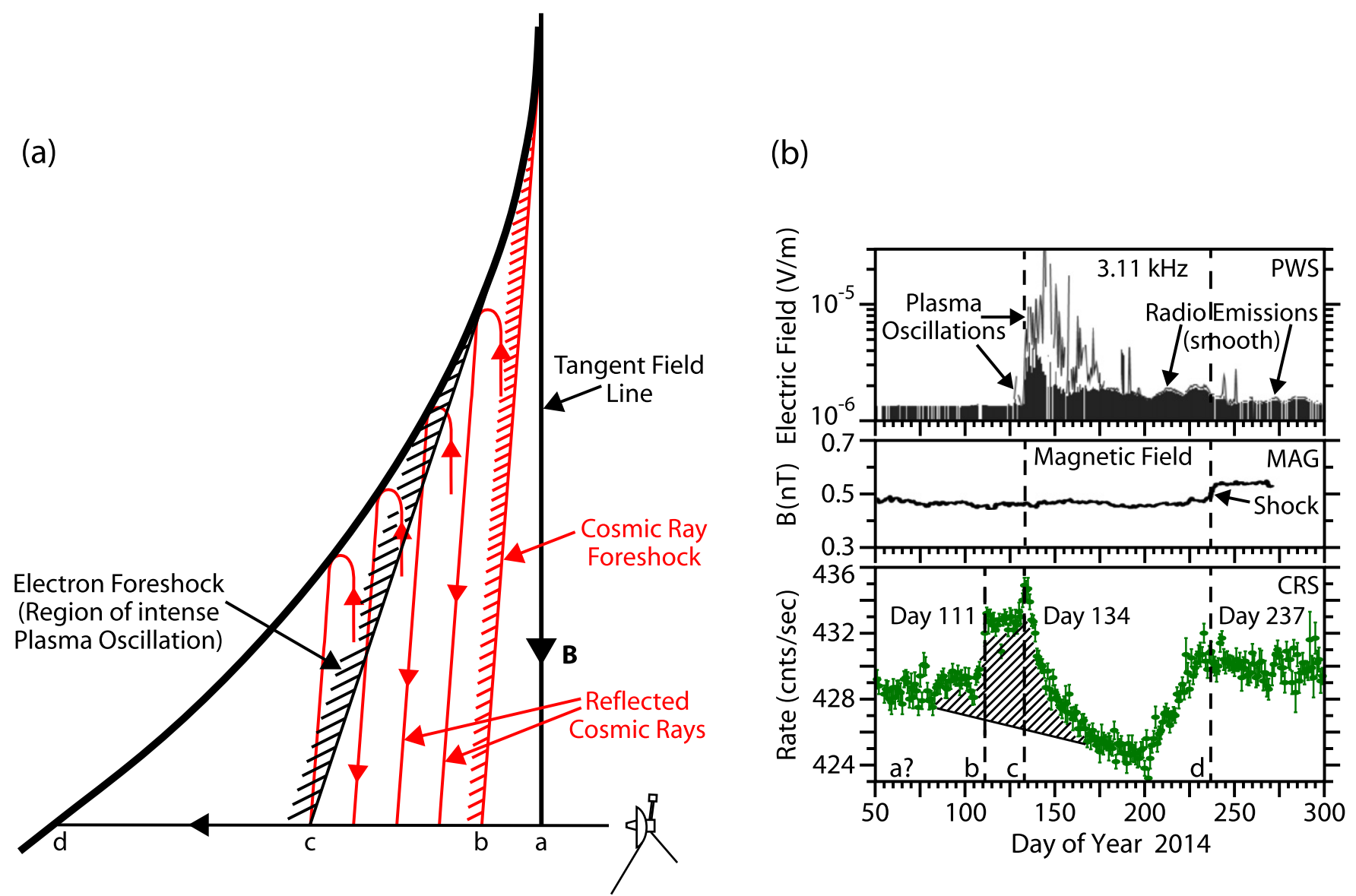

Figure 8. (a) An illustration similar to Figure 7, but showing on a somewhat exaggerated spatial scale how cosmic rays reflected from the shock (red lines) could interact with electron plasma oscillations, which are most intense in the region (black hatching) immediately behind the leading edge of the electron foreshock. (b) The PWS, MAG, and CRS measurements from Figure 3 for the 2014 event, with the points "b," "c," and "d" marked according to the interpretation in illustration (a). There is no clear signature in the data that would tell us where the tangent field line "a" is located, although it must be ahead of the cosmic ray foreshock.

some other mechanism is involved in reflecting the particles from the shock.

There is also another difficulty with the shock reflection model. As we showed earlier, for the 2014 event the intensities of the flat-topped enhancement from days 111 to 134, start to decrease rapidly almost exactly at the onset of the intense electron plasma oscillations, see Figure 3. A similar relationship also occurs for the 2013 event, see Figure 6. Since the energy gain in the reflection process varies smoothly with time after the interstellar magnetic field first contacts the shock, see Figure 2(b) of Jokipii \& Kota (2014), there is no reason that the reflected ion intensity should abruptly increase at the start of the flat-topped enhancement, or to abruptly decrease at exactly the point where the plasma oscillations turn on. Indeed, the abrupt decrease at the onset of the plasma oscillations strongly suggests that the plasma oscillations are somehow involved with the cosmic ray enhancement. Of course it could be that some parameter at the shock that controls the reflected particle intensity, such as the curvature, just happened to change at the onset of the plasma oscillations. However, the fact that the same relationship occurred twice, for two completely independent events, makes this interpretation seem unlikely.

To consider how the plasma oscillations might interact with the cosmic rays, we need to consider how the trajectories of the reflected cosmic rays are related to the leading edge of the electron foreshock, which is where the most intense plasma oscillations occur. This geometry is illustrated in Figure 8(a), which shows the Voyager 1 spacecraft approaching the shock, moving from right to left, as it would in a reference frame fixed to the shock. In this reference frame the spacecraft first crosses the tangent field line at point "a," then the leading edge of the cosmic ray foreshock at point "b," then the leading edge of the electron foreshock at point "c," and finally the shock at point "d." From the diagram one can see that over the entire region from " $b$ " to "c" the cosmic rays reflected from the shock (red lines) must pass through the region of intense plasma oscillations (black hatched region). Thus, for the 2014 event, all of the reflected cosmic rays observed from day 111 to day 134, see Figure 8(b), have been exposed to the intense plasma wave electric fields at some point as they move from the shock to the spacecraft. Therefore, the electric fields of the plasma waves have the potential to cause pitch-angle scattering and energization of these particles. Also, note that after point "c" (day 134 for the 2014 event), the path integrated intensity of the plasma oscillations encountered by the cosmic rays starts to decrease rapidly because of the increasing distance from the leading edge of the electron foreshock where the electric field intensities are most intense. This rapid decrease in the pathintegrated electric field intensity would conveniently explain the rapid decrease in the enhanced cosmic ray intensities after day 134 for the 2014 event. A similar scenario also applies after day 94 for the 2013 event. Qualitatively, the above interaction with the plasma waves provides the correct temporal sequence 
to explain both the onset and decay of the flat-topped cosmic ray enhancement from days 111 to 134 .

So, can we seriously believe that the electron plasma oscillations are causing significant scattering and/or energization of the cosmic rays observed in the region immediately ahead of the shock? In approaching this question we start by noting that the electric field of the plasma oscillations is aligned almost exactly along the magnetic field (Gurnett et al. 2013), which would be quite effective for causing pitch-angle scattering of particles with large, near $90^{\circ}$, pitch angles, such as would be produced by reflection from the shock. However, simple pitch-angle scattering would not explain the sudden decrease in the intensities at point "c," i.e., day 134 for the 2014 event. As discussed earlier, particle acceleration by the plasma wave electric field would nicely account for this feature. In considering these possibilities, we also note that in the interstellar plasma there are exceptionally long path lengths involved, many AU, over which the electric field could act to scatter or energize the cosmic rays. So, we believe that particle acceleration by the plasma wave electric fields is a potentially viable mechanism that merits further study. If on further study the electric field strengths of the plasma oscillations prove to be too small to account for the observed effects, there may be other related possibilities. As we discussed earlier, to generate the upstream radio emission at $f_{\mathrm{p}}$, there must be some lowfrequency plasma wave present in the same region as the plasma oscillations that cannot be detected by the PWS instrument. Such low frequency waves could very well be as important, or even more important than the electron plasma oscillation for scattering or energizing cosmic rays.

Finally, we comment on the V-shaped depression in the intensity of cosmic rays with pitch angles near $90^{\circ}$ between day 0 and day 237 (in 2013) in Figures 3(e) and (f). We have not found any compelling way to explain these depressions in terms of particle reflections at the shock or pitch-angle scattering by the plasma wave electric fields. Although this depression appears to be associated with the region upstream of the shock in the 2014 event, it does not appear to be associated with the shock for the 2013 event or the 2012 event. The reason is that for the 2013 event the depression starts well ahead of any plausible location for the tangent field line, which should be just slightly ahead of the cosmic ray enhancement on day 80, and for the 2012 event the depression starts near the heliopause, which has no obvious connection with the 2012 shock. Based on these arguments we believe that the depression is more likely due to a pre-existing shallow expansion of magnetic field lines draped around the nose of the heliopause, which via conservation of the first adiabatic invariant would act to decrease the cosmic ray intensities, especially near $90^{\circ}$ pitch angles (Cummings et al. 2013; Roelof et al. 2013). In this model the effect of the shock on day 335 for the 2012 event and on day 237 for the 2014 event would be due to reconfiguration of the pre-existing magnetic field via the compression caused by the shock. That a post-shock compression occurred for the 2014 event is clearly indicated by the increase in the plasma density after the passage of the shock, see Figure 5.

The research at the University of Iowa was supported by NASA through contract 1279980 with JPL. The research at
Caltech was supported by NASA contract NNN12AA012, and the research at the Johns Hopkins Applied Physics Laboratory was supported by the Voyager Interstellar Mission under NASA contract NNN06AA01C. The research at the Catholic University of America was supported in part by NASA grant NNX12AC63G, and the research at Goddard Spaceflight Center was supported by NASA contract NNG14PN24P.

\section{REFERENCES}

Anderson, R. R., Parks, G. K., Eastman, T. E., Gurnett, D. A., \& Frank, L. A. 1981, JGR, 86, 4493

Axford, W. I. 1990, in Proc. 1st COSPAR Colloq., Physics of the Outer Heliosphere, ed. S. Grzedzielski \& D. E. Page (Oxford: Pergamon), 7

Bale, S. D., Reiner, M. J., Bougeret, J.-L., et al. 1999, GeoRL, 26, 1573

Burgess, D., Harvey, C. C., Steinberg, J.-L., \& Lacombe, C. 1987, Natur, 330, 732

Burlaga, L. F., \& Ness, N. F. 2014, ApJL, 795, L19

Burlaga, L. F., Ness, N. F., Gurnett, D. A., \& Kurth, W. S. 2013b, ApJL, 778, L3

Burlaga, L. F., Ness, N. F., \& Stone, E. C. 2013a, Sci, 341, 147

Burlaga, L. F., et al. 2005, Sci, 309, 2027

Cairns, I. H. 1987, JGR, 92, 2315

Cairns, I. H., Knock, S. A., Robinson, P. A., \& Kuncic, Z. 2003, SSRv, 107, 27

Cairns, I. H., \& Robinson, P. A. 1992, GeoRL, 19, 2187

Cairns, I. H., \& Zank, G. P. 2001, in COSPAR Colloq. Ser. 11, The Outer Heliosphere: The Next Frontiers, ed. K. Scherer et al. (Oxford: Pergamon), 253

Cairns, I. H., \& Zank, G. P. 2002, GRL, 29, 1143

Cummings, A. C., Stone, E. C., Lai, N., \& Heikkila, B. 2013, in American Geophysical Union Proc. (Washington, D.C.: AGU), SH14B-02

Davis, L. E., Jr. 1955, PhRv, 100, 1440

Decker, R. B. 1983, JGR, 88, 9959

Filbert, P. C., \& Kellogg, P. J. 1979, JGR, 84, 1369

Fitzenreiter, R. J., Klimas, A. J., \& Scudder, J. D. 1984, GRL, 11, 496

Frisch, P. C., Redfield, S., \& Slavin, J. D. 2011, ARA\&A, 49, 237

Fuselier, S. A., \& Cairns, I. H. 2013, ApJ, 771, 83

Gurnett, D. A. 1985, in Collisionless Shocks in the Heliosphere: Reviews of Current Research, Geophysical Monograph 35, ed. B. T. Tsurutani \& R. G. Stone (Washington, DC: American Geophysical Union), 207

Gurnett, D. A., Kurth, W. S., Allendorf, S. C., \& Poynter, R. L. 1993, Sci, 262, 199

Gurnett, D. A., Kurth, W. S., Burlaga, L. F., \& Ness, N. F. 2013, Sci, 341,1489

Gurnett, D. A., Kurth, W. S., \& Scarf, F. L. 1981, Sci, 212, 235

Hoang, S., Fainberg, J., Steinberg, J.-L., Stone, R. G., \& Zwicki, R. H. 1981, JGR, 86, 4531

Jokipii, J. R., \& Kota, J. 2014, ApJL, 794, L4

Krimigis, S. M., Decker, R. M., Roelof, E. C., et al. 2013, Sci, 341, 144

Kuncic, Z., \& Cairns, I. H. 2005, JGR, 110, A07107

Kuncic, Z., Cairns, I. H., Knock, S., \& Robinson, P. A. 2002, GRL, 29, 1161

Kurth, W. S., Gurnett, D. A., McComas, D. J., \& Elliott, H. A. 2014, in American Geophysical Union Proc. (Washington, D.C.: AGU), SH11B-4036

Parker, E. N. 1963, Interplanetary Dynamical Processes (New York: Interscience)

Reiner, M. J., Kaiser, M. L., Fainberg, J., Bougeret, J.-L., \& Stone, R. G. 1998a, GRL, 25, 2493

Reiner, M. J., Kaiser, M. L., Fainberg, J.-L., \& Stone, R. G. 1998b, JGR, 103, 29651

Richardson, J. D., Jasper, J. C., Wang, C., Belcher, J. W., \& Lazarus, A. J. 2008, Natur, 454, 63

Roelof, E. C., Decker, R. B., Krimigis, S. M., \& Hill, M. E. 2013, in American Geophysical Union Proc. (Washington, D.C.: AGU), SH11B-1978

Sauer, K., \& Sydora, R. D. 2012, PPCF, 54, 124045

Scarf, F. L., Fredricks, R. W., Frank, L. A., \& Neugebauer, M. 1971, JGR, 76,5162

Stone, E. C., Cummings, A. C., McDonald, F. B., et al. 2013, Sci, 341, 150

Webber, W. R., \& McDonald, F. B. 2013, GRL, 40, 1665 